\title{
Stochastic reduced order computational model of structures having numerous local elastic modes in low frequency dynamics
}

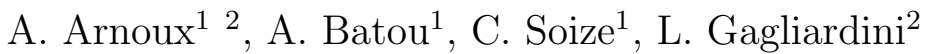 \\ ${ }^{1}$ Université Paris-Est, Laboratoire Modélisation et Simulation \\ Multi-Echelle, MSME UMR 8208 CNRS, 5 Boulevard Descartes, 77454 \\ Marne-la-Vallée, France \\ ${ }^{2}$ PSA Peugeot Citroën, Direction Technique et Industrielle, Centre \\ Technique de Vélizy A, Route de Gisy, 78140 Vélizy Villacoublay, France
}

\begin{abstract}
This paper is devoted to the construction of a stochastic reduced order computational model of structures having numerous local elastic modes in low frequency dynamics. We are particularly interested in automotive vehicles which are made up of stiff parts and flexible components. This type of structure is characterized by the fact that it exhibits, in the low frequency range, not only the classical global elastic modes but also numerous local elastic modes which cannot easily be separated from the global elastic modes. To solve this difficult problem, an innovative method has recently been proposed for constructing a reduced order computational dynamical model adapted to this particular situation for the low frequency range. Then a new adapted generalized eigenvalue problem is introduced and allows a global vector basis to be constructed for the global displacements space. This method requires to decompose the domain of the structure into sub-domains. Such a decomposition is carried out using the Fast Marching Method. This global vec-
\end{abstract}


tor basis is then used to construct the reduced order computational model. Since there are model uncertainties induced by modelling errors in the computational model, the non-parametric probabilistic approach of uncertainties is used and implemented in the reduced order computational model. The methodology is applied to a complex computational model of an automotive vehicle.

Keywords: reduced order model; dynamical analysis; global elastic modes; local elastic modes; fast marching method; uncertainty quantification.

\section{Introduction}

Automotive vehicles are complex three-dimensional dynamical systems for which the prediction of vibration behavior requires highly advanced computational tools. Automotive vehicles are made up of stiff parts such as hollow bodies and flexible parts such as panels and many equipments. The flexible parts of an automotive vehicle as well as equipments are not fully defined during the advanced design phase, which is not the case for the stiff parts. During this draft design, the flexible panels and equipments are not yet completely defined. A vibration analysis in the low frequency (LF) range is performed during the draft design. Since a vibration analysis in the LF range has to be performed in order to assess the advanced design, it is important to construct a robust reduced-order computational model for predicting the LF vibrations of the stiff parts with respect to the design variations of the flexible parts and the equipments, and with respect to uncertainties. The novel approach is used to construct the reduced order model devoted to the prediction of the global displacements on the stiff parts in the LF range. Such 
an unusual reduced order model will be defined as the "Global Reduced Order Model" (G-ROM).

In the present state of the art, reduced-order models can be efficiently constructed using a large number of elastic modes (several thousands) which are calculated with a very large computational model (several millions of DOF). Today, such computation can be done and will be effectively carried out in the application presented in Section 7. As explained above, the objective of this paper is to construct a robust G-ROM with respect to the modifications of the design of the flexible parts of the structure during the advanced design phase. This means that, even if a reduced order model is constructed using a large number of elastic modes for a given structure (that is to say for a given design of the stiff and flexible parts), it is necessary to construct such a robust G-ROM, independently of the computational cost aspects.

Although the noise is radiated by the whole skin of the body, panels are mostly driven by the frame and requirements exist to ensure that no local mode will increase the sound radiation. Unfortunately, during the advance design phase, such requirement are not yet considered and consequently, panel elastic modes often occur in the LF range. Since the G-ROM is defined on the whole mesh, coupling with the cavity will not cause major difficulties, but it was not yet investigated.

In the low frequency range, this type of structure is characterized by the 
fact that it exhibits, not only the classical global elastic modes (involving deflections covering the whole structure), but also numerous local elastic modes (involving localized deflections). In this paper, the terminology "global" in sentences of the type "global elastic mode" or "global displacements" means that we consider displacements fields which are not local displacements in the structure but which are significant at almost all the points of the structural domain. This means that the global displacements are predominant in a displacement field which is identified as a "global displacement". With such a complex heterogeneous structure, the global elastic modes cannot clearly be separated from the local elastic modes because there are many small contributions of the local deformations in the deformations of the global elastic modes and conversely.

The objective of this work is to construct a spatial filtering of elastic modes which have a small wavelength (local elastic modes) in order to only extract the elastic modes which have a large wavelength (global elastic modes). In the literature, there are few studies concerning the global elastic modes extraction when there are mixed with a large number of local elastic modes. In [1], the authors introduced a method to identify the global elastic mode shape only based on geometrical considerations. This method is not really adapted to the finite element computational model. Concerning the construction of reduced order models (ROM) in the low frequency band for automotive vehicles, the common methods are based on the use of modal analysis, sub-structuring techniques and component mode synthesis (see, for instance, $[2,3,4,5,6,7])$. If there is a very large number of local elastic modes in the low frequency band, which cannot easily be separated from the 
global elastic modes, then one obtains a ROM with a very high dimension that is generally the situation encountered for the low frequency dynamical analysis of automotive vehicles.

Considering this particular dynamical behavior of such structures in the LF range, an unusual vector basis of the global displacements space is introduced in order to construct the G-ROM with a small dimension and with the capability to predict the responses on the stiff parts in the LF range. This vector basis is constructed solving an adapted generalized eigenvalue problem.

There are three objectives in this paper: (1) the first one is the construction of a G-ROM with an adapted vector basis, (2) the second one is the construction of a stochastic G-ROM to take into account both the system-parameters uncertainties and the model uncertainties induced by the irreducible errors introduced by neglecting the local displacements contributions and the other modelling errors, (3) the last one concerns the effective construction of the sub-domains.

To achieve the first objective, one reuses the method proposed in [8] which allows a vector basis to be extracted and a G-ROM to be deduced. Concerning the second objective, the stochastic G-ROM is constructed using the nonparametric probabilistic approach [9]. Finally, the third objective is reached using the Fast Marching Method which allows a front to be propagated in a complex mesh of a computational model. Section 3, is devoted to the construction of the reference nominal reduced order model (RN-ROM) obtained as the projection of the reference nominal computational model (RN-CM) on the usual elastic modes. Section 4 deals with the construction of the G-ROM while Section 5 is devoted to the stochastic G-ROM allowing uncertainties to 
be taken into account. Section 6 deals with the construction of sub-domains using the Fast Marching Method (FMM). Finally, Section 7 is devoted to an application related to an automotive vehicle.

\section{Comments concerning notation used}

In this paper, the following notations are used:

1. A lower case Latin or Greek letter is a real deterministic variable (e.g. $\left.x_{1}, u_{1}, q_{1}, \lambda\right)$.

2. A boldface lower case Latin or Greek letter is a real deterministic vector (e.g. $\left.\mathbf{x}, \mathbf{u}, \mathbf{q}, \boldsymbol{\varphi}_{1}\right)$.

3. An upper case Latin "blackboard bold" letter is a real vector (e.g. $\mathbb{U}, \mathbb{F})$.

4. An upper case Latin letter is a real random variable (e.g. $Q$ ).

5. A boldface upper case Latin letter is a real random vector (e.g. $\mathbf{U})$.

6. An upper case Latin or Greek or "blackboard bold" letter between brackets is a real deterministic matrix (e.g. $[A],[\Phi],[M]$ ).

7. A boldface upper case Latin letter between brackets is a real random matrix (e.g. $[\mathbf{M}])$.

\section{Reference nominal computational model (RN-CM)}

We are interested in predicting, in the frequency band of analysis $\mathcal{B}=$

$\left[\omega_{\min }, \omega_{\max }\right]$ with $0<\omega_{\min }$, the frequency response functions of a fixed damped structure occupying a bounded domain $\Omega$ and referenced in a Cartesian system of coordinates. We mean by "fixed structure", a deformable structure 
for which there are no rigid body displacements due to locked displacements on a part of its boundary. If the damped structure is not fixed, that is to say, if there are rigid body displacements ( 1 to 6 depending on the locked body displacements, and equal to 6 for a completely free structure), then the proposed theory can straightforwardly be applied. The rigid body modes then belong to the basis of the global displacements which will be introduced in Section 4. Let $\mathbf{x}=\left(x_{1}, x_{2}, x_{3}\right)$ be a point in $\Omega$. For all $\omega$ fixed in $\mathcal{B}$, the complex vector-valued displacement field is denoted by $\mathbf{u}(\mathbf{x}, \omega)=\left(u_{1}(\mathbf{x}, \omega), u_{2}(\mathbf{x}, \omega), u_{3}(\mathbf{x}, \omega)\right)$. The computational model of the structure is constructed using the Finite Element (FE) method (see [10]). Let $\mathbb{U}(\omega)$ be the complex vector of the $m$ degrees of freedom (DOF) discretizing the displacement field. Let $[\mathbb{M}],[\mathbb{D}]$ and $[\mathbb{K}]$ be the positive-definite symmetric $(m \times m)$ mass, damping and stiffness matrices. The vector of the discretized external forces is denoted by $\mathbb{F}(\omega)$. For fixed $\omega$, the complex vector $\mathbb{U}(\omega)$ is the solution of the following complex matrix equation,

$$
\left(-\omega^{2}[\mathbb{M}]+\mathrm{i} \omega[\mathbb{D}]+[\mathbb{K}]\right) \mathbb{U}(\omega)=\mathbb{F}(\omega)
$$

The eigenvalues $\lambda_{\alpha}=\omega_{\alpha}^{2}$ (square of the eigenfrequency $\omega_{\alpha}$ ) and the associated elastic modes $\varphi_{\alpha}$ of the conservative part of the dynamical computational model are the solution of the following generalized eigenvalue problem,

$$
[\mathbb{K}] \varphi_{\alpha}=\lambda_{\alpha}[\mathbb{M}] \varphi_{\alpha}
$$

An approximation at order $n$ with $n \ll m$, of the solution $\mathbb{U}(\omega)$ of Eq. (1), can classically be constructed using modal analysis,

$$
\mathbb{U}(\omega) \simeq \sum_{\alpha=1}^{n} q_{\alpha}(\omega) \boldsymbol{\varphi}_{\alpha}=[\Phi] \mathbf{q}(\omega)
$$


in which $\mathbf{q}(\omega)=\left(q_{1}(\omega), \ldots, q_{n}(\omega)\right)$ is the complex vector of the $n$ generalized coordinates and where $[\Phi]=\left[\boldsymbol{\varphi}_{1} \ldots \boldsymbol{\varphi}_{n}\right]$ is the $(m \times n)$ real matrix of the elastic modes associated with the $n$ first eigenvalues.

The "reference nominal computational model (RN-CM)" is defined by Eq. (1) as the computational model for which the parameters are fixed to their nominal values. In practice, the $\mathrm{RN}-\mathrm{CM}$ corresponds to a large finite element model with numerous degrees-of-freedom (DOF). Consequently, the solution of the RN-CM can classically be approximated using the modal analysis with a large number of elastic modes in order that convergence of the approximation be reached. This approximation at order $n$, which is constructed solving the "reference nominal reduced order model" (RN-ROM) defined by Eq. (3), is then considered as the solution of the RN-CM.

\section{Construction of the G-ROM}

In this section, we summarize the method introduced in [8] in which theoretical details can be found. In addition, one presents complements concerning the damping modelling for the low frequency band. This method allows an adapted basis of the global displacements and a basis of the local displacements to be constructed by solving two separated generalized eigenvalue problems. It should be noted that these two bases are not made up of the elastic modes. This method is based on the construction of a projection operator which introduces a kinematic reduction of the displacement field for calculating the kinetic energy, while the elastic energy remains exact. A adapted partition of domain $\Omega$ in sub-domains is introduced and allows the 
projection operator to be constructed.

\subsection{Projection operator and projection of the mass matrix}

In this section, we introduce a projection operator and the projection of the mass matrix which allows the global displacements basis and a local displacements basis to be computed. Domain $\Omega$ is decomposed into $N$ non overlapped sub-domains $\Omega_{j}$ with $j=1, \ldots, N$. For $j$ equal to $1, \ldots, N$,

$$
\Omega=\bigcup_{j=1}^{N} \Omega_{j} .
$$

The construction of such subdomains is very difficult for complex geometry of domain $\Omega$ (such that for an automotive vehicle) and requires specific methods which will be detailed in Section 6 . Let $\mathbf{u} \mapsto h^{r}(\mathbf{u})$ be the projection operator defined by

$$
\left\{h^{r}(\mathbf{u})\right\}(\mathbf{x})=\sum_{j=1}^{N} \mathbb{1}_{\Omega_{j}}(\mathbf{x}) \frac{1}{m_{j}} \int_{\Omega_{j}} \rho\left(\mathbf{x}^{\prime}\right) \mathbf{u}\left(\mathbf{x}^{\prime}\right) d \mathbf{x}^{\prime},
$$

in which $\mathbf{x} \mapsto \mathbb{1}_{\Omega_{j}}(\mathbf{x})=1$ if $\mathbf{x}$ is in $\Omega_{j}$ and $=0$ otherwise. For all $j=1, \ldots, N$,

the local mass $m_{j}$ is defined by $m_{j}=\int_{\Omega_{j}} \rho(\mathbf{x}) d \mathbf{x}$, where $\mathbf{x} \mapsto \rho(\mathbf{x})$ is the mass density. Let $\left[H^{r}\right]$ be the $(m \times m)$ matrix of the projection operator $h^{r}$ relative to the finite element basis of the computational model. The vector $\mathbb{U}$ can be written as $\mathbb{U}=\mathbb{U}^{r}+\mathbb{U}^{c}$ in which

$$
\begin{gathered}
\mathbb{U}^{r}=\left[H^{r}\right] \mathbb{U} \\
\mathbb{U}^{c}=\left[H^{c}\right] \mathbb{U}=\mathbb{U}-\mathbb{U}^{r},
\end{gathered}
$$

in which $\left[H^{c}\right]=\left[I_{m}\right]-\left[H^{r}\right]$ where $\left[I_{m}\right]$ is the $(m \times m)$ identity matrix. Then, the projected $(m \times m)$ mass matrix, $\left[\mathbb{M}^{r}\right]$, is defined by

$$
\left[\mathbb{M}^{r}\right]=\left[H^{r}\right]^{T}[\mathbb{M}]\left[H^{r}\right]
$$


and the complementary $(m \times m)$ mass matrix $\left[\mathbb{M}^{c}\right]$ is defined by

$$
\left[\mathbb{M}^{c}\right]=\left[H^{c}\right]^{T}[\mathbb{M}]\left[H^{c}\right]
$$

Using the properties of the projection operator defined by Eq. (5), it can be shown [8] that

$$
\left[\mathbb{M}^{c}\right]=[\mathbb{M}]-\left[\mathbb{M}^{r}\right]
$$

It should be noted that Eq. (5) corresponds to the theory of 3D continuous media. In practice, the computational model of a complex structure such as an automotive vehicle contains many types of finite elements such as spring, beam, plate, shells, 3D solid elements. Consequently, there are translation and rotation DOFs. For a sufficiently fine finite element mesh (that is the case for the mesh of an automotive vehicle), the averaging of the rotation fields over each subdomain does not induce a significant effect with respect to the translation field as used in Eq. (5). Consequently, an efficient approximation consists in setting to zero the elements in the matrix $\left[\mathrm{H}^{r}\right]$ corresponding to rotation DOFs.

\subsection{Construction of vector bases for the global and the local displacements}

Two methods can be used to calculate the global displacements vector basis that will be used to reduce the matrix equation for the low frequency band: the direct method and the double projection method. For the direct method, the vector basis of the global displacements is directly calculated using the projection of mass matrix $[\mathbb{M}]$. We then have to solve the following generalized eigenvalue problem,

$$
[\mathbb{K}] \boldsymbol{\phi}^{g}=\lambda^{g}\left[\mathbb{M}^{r}\right] \boldsymbol{\phi}^{g}
$$


in which $\lambda^{g}$ is the eigenvalue and $\phi^{g}$ is the associated global displacements eigenvector. The number of subdomains is N. The projection operator yields a projected mass matrix (see Eq. (8)) whose rank is $3 \mathrm{~N}$ (3translations). So the generalized eigenvalue problem defined by Eq. ( 11) corresponds to a mechanical system with $3 \mathrm{~N}$ dynamical DOFs and consequently, the maximum number of global displacements eigenvectors is $3 \mathrm{~N}$.

It is also proven [8] that a local displacement vector basis, which will not be used to construct the reduced order model in the low frequency band, can be obtained solving the following generalized eigenvalue problem,

$$
[\mathbb{K}] \phi^{\ell}=\lambda^{\ell}\left[\mathbb{M}^{c}\right] \phi^{\ell}
$$

where $\phi^{\ell}$ is an local displacements eigenvector.

The double projection method [8], which will be used in this work, is less intrusive with respect to the commercial software and less time-consuming than the direct method. This method consists in approximating $\phi^{g}$ and $\phi^{\ell}$, using Eq. (3), by $\widetilde{\phi}^{g}$ and $\widetilde{\phi}^{\ell}$ such that

$$
\phi^{g}=[\Phi] \widetilde{\phi}^{g} \quad, \quad \phi^{\ell}=[\Phi] \widetilde{\phi}^{\ell} .
$$

Then Eqs. (13), (11) and (12) yields

$$
[\widetilde{K}] \widetilde{\phi}^{g}=\lambda^{g}\left[\widetilde{M}^{r}\right] \widetilde{\phi}^{g} \quad, \quad[\widetilde{K}] \widetilde{\phi}^{\ell}=\lambda^{\ell}\left[\widetilde{M}^{c}\right] \widetilde{\phi}^{\ell}
$$

in which $\left[\widetilde{M}^{r}\right]=\left[\Phi^{r}\right]^{T}[\mathbb{M}]\left[\Phi^{r}\right]$ and $[\widetilde{K}]=[\Phi]^{T}[\mathbb{K}][\Phi]$. In this equation, $\left[\Phi^{r}\right]$ is the $(m \times n)$ real matrix such that $\left[\Phi^{r}\right]=\left[H^{r}\right][\Phi]$. One has $\left[\widetilde{M}^{c}\right]=$ $\left[\Phi^{c}\right]^{T}[\mathbb{M}]\left[\Phi^{c}\right]$ in which $\left[\Phi^{c}\right]$ is an $(m \times n)$ real matrix such that $\left[\Phi^{c}\right]=$ 
$\left[H^{c}\right][\Phi]=[\Phi]-\left[\Phi^{r}\right]$. It can be proven $[8]$ that the family $\left\{\boldsymbol{\phi}_{1}^{g}, \ldots, \boldsymbol{\phi}_{3 N}^{g}\right.$, $\left.\phi_{1}^{\ell}, \ldots, \phi_{m-3 N}^{\ell}\right\}$ is a vector basis of $\mathbb{R}^{m}$. The projection of $\mathbb{U}(\omega)$ on this family of real vectors associated with the $n_{g}$ first global displacements eigenvectors such that $n_{g} \leq 3 N$ and with the $n_{\ell}$ first local displacements eigenvectors such that $n_{\ell} \leq m-3 N$ is written as

$$
\mathbb{U}^{\left(n_{g}, n_{\ell}\right)}(\omega)=\sum_{\alpha=1}^{n_{g}} q_{\alpha}^{g}(\omega) \phi_{\alpha}^{g}+\sum_{\beta=1}^{n_{\ell}} q_{\beta}^{\ell}(\omega) \phi_{\beta}^{\ell} .
$$

It should be noted that, for the double projection method, $n$ must be such that $n_{g}+n_{\ell} \leq n$.

\subsection{Global reduced order model}

In this paper, the G-ROM is constructed using only the global displacements vector basis introduced in Section 4.2. Then, the approximation $\mathbb{U}^{\left(n_{g}\right)}(\omega)$ of $\mathbb{U}(\omega)$ is defined by

$$
\mathbb{U}^{\left(n_{g}\right)}(\omega)=\sum_{\alpha=1}^{n_{g}} q_{\alpha}^{g}(\omega) \phi_{\alpha}^{g}
$$

It should be noted that, in general, $\mathbb{U}^{(3 N)}(\omega)$ is not equal to $\mathbb{U}(\omega)$ because the family $\left\{\phi_{1}^{g}, \ldots, \phi_{3 N}^{g}\right\}$, which is a vector basis of the global displacements, is not a vector basis of $\mathbb{R}^{m}$. Nevertheless, because we are interested in constructing the G-ROM to predict the global displacements responses on the stiff parts in the low frequency band, the $3 N$ vector basis of the global displacements is sufficient to give a good approximation of $\mathbb{U}(\omega)$ for $\omega$ in $\mathcal{B}$. In addition and in general, it is not necessary to use the $3 N$ vectors of the basis but only $n_{g}<3 N$. In this condition, the convergence of $\mathbb{U}^{\left(n_{g}\right)}(\omega)$ can be analysed for $n_{g}$ going to $3 N$. Nevertheless, approximation $\mathbb{U}^{\left(n_{g}\right)}(\omega)$ converges 
to response $\mathbb{U}(\omega)$ if $3 N$ goes to $m$, that is to say if the number $N$ of subdomains increased and then if the size of subdomains goes to zero. Then, the efficiency of the method directly depends on the domain decomposition in subdomains which will be carried out in order to obtain a small and accurate G-ROM to predict the global displacements responses in $\mathcal{B}$. Using Eq. (1), we obtain the following G-ROM defined by Eq. (16) and by the following matrix equation,

$$
\left(-\omega^{2}\left[M^{g g}\right]+\mathrm{i} \omega\left[D^{g g}\right]+\left[K^{g g}\right]\right) \mathbf{q}^{g}(\omega)=\mathbf{f}^{g}(\omega),
$$

where $\left[M^{g g}\right]=\left[\Phi^{g}\right]^{T}[\mathbb{M}]\left[\Phi^{g}\right],\left[D^{g g}\right]=\left[\Phi^{g}\right]^{T}[\mathbb{D}]\left[\Phi^{g}\right],\left[K^{g g}\right]=\left[\Phi^{g}\right]^{T}[\mathbb{K}]\left[\Phi^{g}\right]$, and $\mathbf{f}^{g}=\left[\Phi^{g}\right]^{T} \mathbb{F}$, in which $\left[\Phi^{g}\right]=\left[\boldsymbol{\phi}_{1}^{g} \ldots \boldsymbol{\phi}_{n_{g}}^{g}\right]$ is the $\left(m \times n_{g}\right)$ real matrix of the global displacements eigenvectors.

It should be noted that the global displacements eigenvectors are not orthogonal to the local displacements eigenvectors with respect to the mass and stiffness matrices. Consequently there is a coupling between the generalized coordinates $\mathbf{q}^{g}$ and $\mathbf{q}^{\ell}$. In these coordinates, there is a transfer of the mechanical energy from $\mathbf{q}^{g}$ to $\mathbf{q}^{\ell}$, and at the equilibrium, the mechanical energy stored by $\mathbf{q}^{\ell}$ induces an apparent damping for the coordinates $\mathbf{q}^{g}[2]$.

In order to take into account the presence of this apparent damping, in Eq. (17), the reduced damping matrix $\left[D^{g g}\right]$ is replaced by a modified damping matrix $\left[D^{g g, \text { mod}}\right]$. This modified matrix is identified in minimizing the distance between the response given by the G-ROM and the response given by the RN-CM. Since $\left[D^{g g, \text { mod }}\right]$ is a full positive-definite $\left(n_{g} \times n_{g}\right)$ real matrix, such a direct identification would correspond to a high dimensional inverse problem in the set of all the positive-definite $\left(n_{g} \times n_{g}\right)$ real matrices, which 
would be difficult to solve. One then introduces a parametric representation of matrix $\left[D^{g g}\right]$ with $n_{g}$ unknown parameters $a_{1}, \ldots, a_{n_{g}}$. Let $[A]$ be the diagonal matrix such that $[A]_{j j}=a_{j}$. The Cholesky decomposition of matrix $\left[D^{g g}\right]$ is written as $\left[D^{g g}\right]=\left[L^{g g}\right]\left[L^{g g}\right]^{T}$. The matrix $\left[D^{g g, \text { mod }}\right]$ is then constructed as follows

$$
\left[D^{g g, \bmod }\right](\mathbf{a})=\left[L^{g g}\right][A]\left[L^{g g}\right]^{T},
$$

in which a denotes the vector of components $a_{1}, \ldots, a_{n_{g}}$. Consequently, for $n_{g}$ fixed, the identification of matrix $\left[D^{g g \text {,mod }}\right]$ consists in identifying vector

a. One then have to solve the following optimisation problem

$$
\mathbf{a}^{\mathrm{opt}}=\arg \min _{a_{1}>0, \ldots, a_{n_{g}}>0} \int_{\mathcal{B}}\left\|\mathbb{U}(\omega)-\mathbb{U}_{\bmod }(\omega ; \mathbf{a})\right\|^{2} d \omega,
$$

in which $\|$.$\| is the Hermitian norm, where \mathbb{U}(\omega)$ is the reference solution calculated with Eq. (1) and where $\mathbb{U}_{\bmod ; \mathbf{a}}(\omega)$ is the solution of the following modified G-ROM,

$$
\begin{gathered}
\mathbb{U}_{\bmod }(\omega ; \mathbf{a})=\sum_{\alpha=1}^{n_{g}} q_{\alpha}^{g, \bmod }(\omega ; \mathbf{a}) \boldsymbol{\phi}_{\alpha}^{g}, \\
\left(-\omega^{2}\left[M^{g g}\right]+\mathrm{i} \omega\left[D^{g g, \bmod }(\mathbf{a})\right]+\left[K^{g g}\right]\right) \mathbf{q}^{g, \bmod }(\omega ; \mathbf{a})=\mathbf{f}^{g}(\omega) .
\end{gathered}
$$

\section{Probabilistic model of uncertainties for the G-ROM and identi- fication}

In this section, a stochastic model of uncertainties is constructed for the G-ROM defined by Eqs. (20) and (21). Both the system-parameters uncertainties and the model uncertainties induced by modelling errors are taken into account using the non-parametric probabilistic approach of uncertainties 
(see [9]). This method consists in replacing the generalized mass, damping and stiffness matrices in Eq. (21) by random matrices. In the G-ROM defined by Eqs. (20) and (21), a part of the modelling errors are induced by the local displacements which are not taken into account. Another part of the modelling errors is due to the $\mathrm{RN}-\mathrm{CM}$ which is a representation of a very complex mechanical system such as an automotive vehicle. The main objective of this work is to validate the stochastic G-ROM, including the stochastic model of uncertainties, in order to predict the response on the stiff parts in the low frequency range. The non-parametric probabilistic model of uncertainties has been identified and validated for automotive vehicle in $[11,12,13]$. In particular, it has been proven that the confidence regions of the random responses are not really sensitive to the damping uncertainties. In this work, one reuses these results to construct a reference solution which then includes uncertainties only for the mass and stiffness parts. The nonparametric probabilistic model of uncertainties is implemented in the RNROM. The dispersion parameters controlling uncertainties, for the random mass matrix and the random stiffness matrix, are $\delta_{M}^{\mathrm{ref}}$ and $\delta_{K}^{\mathrm{ref}}$ and have been identified in [11] for automotive vehicles using experimental measurements. In this section, one identifies the stochastic G-ROM, using the random response $\mathbf{U}^{\text {ref }}(\omega)$ of the stochastic RN-ROM.

\subsection{Construction of the stochastic G-ROM}

As explained above, uncertainties must be taken into account in the GROM defined by Eqs. (20) and (21). Consequently, matrices $\left[M^{g g}\right]$ and $\left[K^{g g}\right]$ are replaced [9] by the random matrices $\left[\mathbf{M}^{g g}\left(\delta_{M^{g g}}\right)\right]$ and $\left[\mathbf{K}^{g g}\left(\delta_{K^{g g}}\right)\right]$ which 
depend on the parameters $\delta_{M^{g g}}$ and $\delta_{K^{g g}}$. These parameters must be identified using the response $\mathbf{U}^{\mathrm{ref}}(\omega)$ and the maximum likelihood method. The stochastic G-ROM is then written as

$$
\begin{gathered}
\mathbf{U}^{g}(\omega)=\sum_{\alpha=1}^{n_{g}} Q_{\alpha}^{g}(\omega) \phi_{\alpha}^{g}, \\
\left(-\omega^{2}\left[\mathbf{M}^{g g}\left(\delta_{M^{g g}}\right)\right]+\mathrm{i} \omega\left[D^{g g, \bmod }\left(\mathbf{a}^{\mathrm{opt}}\right)\right]+\left[\mathbf{K}^{g g}\left(\delta_{K^{g g}}\right)\right]\right) \mathbf{Q}^{g}(\omega)=\mathbf{f}^{g}(\omega) .
\end{gathered}
$$

5.2. Identification of the stochastic G-ROM using the response of the stochastic $R N-R O M$

It is assumed that $n_{\text {obs }}$ degrees of freedom (dof) are observed for $n_{\text {freq }}$ frequencies $\omega_{1}, \ldots, \omega_{n_{\text {freq }}}$ in the frequency band of analysis $\mathcal{B}$. The random observation vector $\mathbf{W}^{\mathrm{obs}}(\omega)=\left(\log _{10}\left|U_{1}^{g}\left(\omega_{1}\right)\right|, \ldots, \log _{10}\left|U_{1}^{g}\left(\omega_{n_{\text {freq }}}\right)\right|, \ldots\right.$, $\left.\log _{10}\left|U_{n_{\text {obs }}}^{g}\left(\omega_{1}\right)\right|, \ldots, \log _{10}\left|U_{n_{\text {obs }}}^{g}\left(\omega_{n_{\text {freq }}}\right)\right|\right)$ is introduced. The corresponding reference observation vector is denoted by $\mathbf{W}^{\text {ref }}$. Let $\mathbf{w}^{\text {ref, } 1}, \ldots, \mathbf{w}^{\text {ref, } n_{\exp }}$ be $n_{\exp }$ independent realizations of $\mathbf{W}^{\text {ref }}$ calculated using the stochastic reference computational model. One introduces the admissible domain $\mathcal{C}_{\text {ad }}$ of the vector $\boldsymbol{\delta}=\left(\delta_{M^{g g}}, \delta_{K^{g g}}\right)$. The maximum likelihood method yields

$$
\boldsymbol{\delta}^{\text {opt }}=\arg \max _{\boldsymbol{\delta} \in \mathcal{C}_{\text {ad }}} \mathcal{L}(\boldsymbol{\delta}) \quad, \quad \mathcal{L}(\boldsymbol{\delta})=\sum_{j=1}^{n_{\text {exp }}}\left\{\log \left(p_{\mathbf{W}^{\text {obs }}}\left(\mathbf{w}^{\mathrm{ref}, j} ; \boldsymbol{\delta}\right)\right\},\right.
$$

in which $p_{\mathbf{W}^{\text {obs }}}\left(\mathbf{w}^{\mathrm{ref}, j} ; \boldsymbol{\delta}\right)$ is the value of the probability density function of random vector $\mathbf{W}^{\text {obs }}$ for $\mathbf{w}=\mathbf{w}^{\text {ref }, j}$.

The optimization problem, defined by Eq. (24), is performed for the identification of vector $\boldsymbol{\delta}=\left(\delta_{M^{g g}}, \delta_{K^{g g}}\right)$ which belongs to an admissible set $\mathcal{C}_{\mathrm{ad}}=\left[a_{M^{g g}}, b_{M^{g g}}\right] \times\left[a_{K^{g g}}, b_{K^{g g}}\right]$. The Monte Carlo method is used to solve 
the stochastic Eq. (23). Such a solver is very fast because the stochastic G-ROM has generally a small dimension (the value of $n_{g}$ is a few tens). The algorithm used is the following.

(i) A statistical reduction of the random observation vector $\mathbf{W}^{\text {obs }}$ (which is in high dimension, several thousands) is performed using a principal component analysis. The statistical reduced random observation vector is then a new random vector denoted by $\mathbf{W}_{\text {red }}^{\text {obs }}$ in low dimension (a few tens or one hundred).

(ii) The components of $\mathbf{W}_{\text {red }}^{\text {obs }}$ are uncorrelated (but statistically dependent) random variables. An approximation of the log-likelihood function is used consisting in replacing the joint pdf of the random vector $\mathbf{W}_{\text {red }}^{\text {obs }}$ by the product of the pdf of its random components. Then, the one-dimensional Gaussian kernel estimation method is used for estimating the value of the onedimension pdf, at the experimental points, of each random components.

(iii) Since there are only two scalar parameters, the trial method can be used as an optimization algorithm. Since the cost function varies slowly on domain $\mathcal{C}_{\mathrm{ad}}$, a coarse Cartesian mesh of $\left[a_{M^{g g}}, b_{M^{g g}}\right] \times\left[a_{K^{g g}}, b_{K^{g g}}\right]$ is introduced for the trial method.

This approach yields a good and efficient approximation for the estimation of the optimal value of $\delta_{M^{g g}}$ and $\delta_{K^{g g}}$. 


\section{Implementation of the Fast Marching Method for constructing the subdomains}

In this section, a methodology is proposed to construct of the subdomains introduced in Section 4.1. The decomposition of domain $\Omega$ is carried out using the mesh of the FE model. One then has to construct the groups of nodes using the connectivities. For structures with complex curved geometry, such a task is not easy to be carried out. There are several types of finite elements in the computational model(3D, 2D, 1D and 0D elements). The method one proposes for the decomposition of domain $\Omega$ in subdomains, is based on the use of the FMM introduced in [14] which provides a method to propagate a front (the notion of front will be defined below) on connected parts from a starting point. In this section, the FMM is summarized and is used to construct the subdomains.

\subsection{Presentation of the Fast Marching Method (FMM)}

Let $\mathbf{x}_{0}$ be a fixed point belonging to $\Omega$. Let $g(\mathbf{x})$ be the geodesic distance, between a point $\mathbf{x}$ and the point $\mathbf{x}_{0}$. It should be noted that for a $3 \mathrm{D}$ volume, the geodesic distance coincide with the Euclidean distance $\left\|\mathbf{x}-\mathbf{x}_{0}\right\|$ while for a 2D curved surface, the geodesic distance between two points on the surface is the length of the shortest distance between these two points in staying on the surface. A front related to $\mathbf{x}_{0}$ is defined as the subset of all the points $\mathbf{x}$ in $\Omega$ such that $g(\mathbf{x})$ has a fixed value. The FMM [14] allows the front to be propagated in the mesh from starting point $\mathbf{x}_{0}$. One then has to calculate $g(\mathbf{x})$ verifying the following non-linear Eikonal equation

$$
\|\nabla g(\mathbf{x})\|=s(\mathbf{x}) \quad, \quad \mathbf{x} \in \Omega
$$


in which $\nabla$ is the gradient with respect to $\mathbf{x}$, and where $s(\mathbf{x})$ is a given arbitrary positive-valued function. The boundary condition associated with Eq. (25), is written as $g(\mathbf{x})=0$ on $\Gamma_{0}$ which is a curved line or a surface containing $\mathbf{x}_{0}$. Using the finite element mesh of $\Omega$, Eq. (25) is discretized using an upwind approximation (forward finite difference) for the gradient.

\subsubsection{Simple case for explaining the methodology}

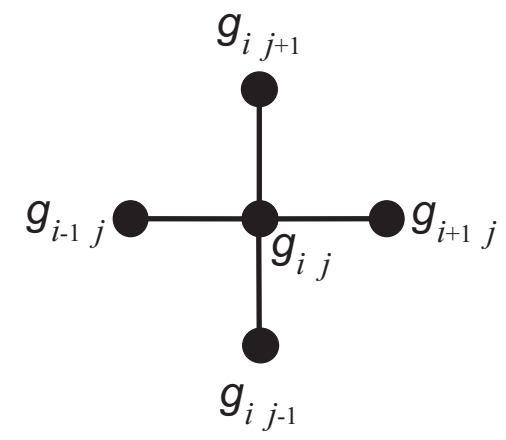

Figure 1: Simple case: spatial discretisation for the Eikonale equation.

In order to simply explain the FMM, we consider a Cartesian mesh of a plane square domain. Each finite element mesh is square. The mesh size is $h$ and the nodes of the finite element mesh are denoted by $\mathbf{x}_{i j}$. One then has to find $g_{i j}=g\left(\mathbf{x}_{i j}\right)$ satisfying the following equation (see Fig. 1)

$$
\begin{aligned}
& \left\{\max \left(g_{i j}-g_{i-1 j}, g_{i j}-g_{i+1}, 0\right)\right\}^{2} \\
& \quad+\left\{\max \left(g_{i j}-g_{i j-1}, g_{i j}-g_{i j+1}, 0\right)\right\}^{2}=h^{2} s_{i j}^{2} .
\end{aligned}
$$

Since function $s(\mathbf{x})$ can arbitrarily be chosen, $s_{i j}$ can be taken at 1 for all $i$ and $j$. In Eq. (26), the information propagates in a unique way. This equation allows the front to be propagated from the starting point. The use 
of the word Fast in FMM is due to the fact that the value $g_{i j}$ of function $g(\mathbf{x})$ at node $\mathbf{x}=\mathbf{x}_{i j}$ is calculated with Eq. (26) which corresponds to the spatial discretization of Eq. (25) related to the Euclidean norm of the gradient of function $g$ and consequently, FMM uses only the nodes belonging to a small domain (called a Narrow Band (NB) domain). In the FMM, the algorithm introduces three groups of nodes:

(1) Alive node $\mathbf{x}_{i j}$ for which the value of $g_{i j}$ is fixed and does not change,

(2) Trial node $\mathbf{x}_{i j}$ for which the value of $g_{i j}$ is given but has to be updated until it becomes an alive node. The set of all the trial nodes constitutes the Narrow Band,

(3) Far node $\mathbf{x}_{i j}$ which has not been reached by the front and therefore is such that $g_{i j}=+\infty$.

The front is propagated using the following algorithm:

\section{Initialization}

- Choose a starting node $\mathbf{x}_{0}$, rewritten as $\mathbf{x}_{00}$, which is an alive node and set $g_{00}=g\left(\mathbf{x}_{00}\right)=0$ (see Fig. $\left.2-(\mathrm{a})\right)$.

- The four neighbouring nodes $\mathbf{x}_{i j}$ of $\mathbf{x}_{00}$ become trial nodes for which $g_{i j}=h s_{00}$.

- All the other nodes $\mathbf{x}_{k \ell}$ are far nodes for which $g_{k \ell}=+\infty$.

Loop

- Search among trial nodes, the node $\mathbf{x}_{i j}$ with the smallest value of $g_{i j}$.

- Remove $\mathbf{x}_{i j}$ from trial nodes and add $\mathbf{x}_{i j}$ to alive nodes (see Fig. 2-(b)). 
- For each neighbouring node $\mathbf{x}_{k \ell}$ of node $\mathbf{x}_{i j}$, there are two cases:

- if the neighbouring node is a far node, then add it to trial nodes with $g_{k \ell}=g_{i j}+h s_{i j}$.

- if the neighbouring node is a trial node, then the value of $g_{k \ell}$ is updated solving Eq. (26).

The Loop is repeated until all the nodes are alive nodes (see Fig. 2-(d)).

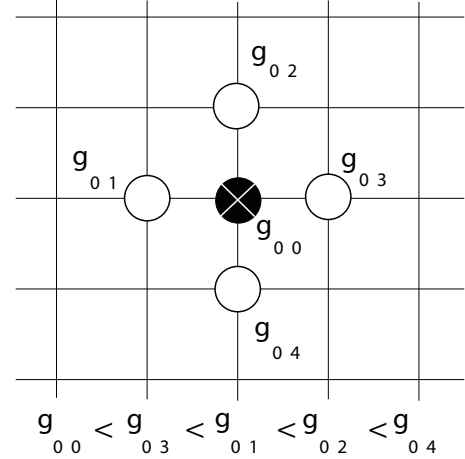

(a)



(c)

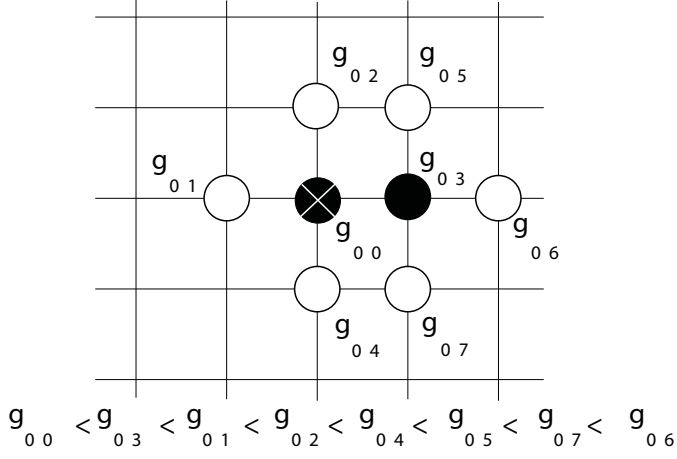

(b)



(d)

Figure 2: Diagram of the Fast Marching Method: step 1 (a), step 2 (b), step 3 (c) and last step corresponding to the diagram, step 5 (d). 


\subsubsection{Extension of the explanation for a triangular mesh}

For triangular meshes, the algorithm described above is adapted. Let us consider the case presented in Fig. 3 for which the value $g_{c}=g\left(\mathbf{x}_{c}\right)$ of $g$ for node $\mathbf{x}_{c}$ is updated using the value $g_{a}=g\left(\mathbf{x}_{a}\right)$ of node $\mathbf{x}_{a}$ and the value $g_{b}=g\left(\mathbf{x}_{b}\right)$ of node $\mathbf{x}_{b}$, which are alive nodes. We then obtain the following

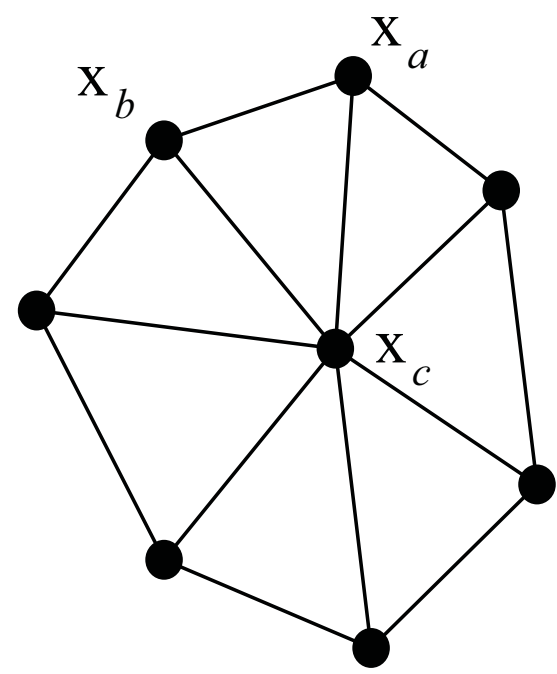

Figure 3: Triangular mesh (with acute angle) around node $\mathbf{x}_{c}$.

equation

$$
\left(\mathbf{a}^{T}[Q] \mathbf{a}\right) g_{c}^{2}+\left(2 \mathbf{a}^{T}[Q] \mathbf{b}\right) g_{c}+\left(\mathbf{b}^{T}[Q] \mathbf{b}\right)=s_{c}^{2}
$$

in which $\mathbf{a}=(1,1), \mathbf{b}=\left(-g_{a},-g_{b}\right)$ and where the matrix $[Q]$ is written as $[Q]=\left([P][P]^{T}\right)^{-1}$ in which the matrix $[P]$ is defined by

$$
[P]=\left[\begin{array}{ccc}
x_{c}-x_{a} & y_{c}-y_{a} & z_{c}-z_{a} \\
x_{c}-x_{b} & y_{c}-y_{b} & z_{c}-z_{b}
\end{array}\right],
$$

in which $\left(x_{a}, y_{a}, z_{a}\right),\left(x_{b}, y_{b}, z_{b}\right)$ and $\left(x_{c}, y_{c}, z_{c}\right)$ are the coordinates of points $\mathbf{x}_{a}, \mathbf{x}_{b}$ and $\mathbf{x}_{c}$. It can be shown that Eq. (27) admits two positive solutions 
if the angle $\widehat{\mathbf{x}_{a} \mathbf{x}_{c} \mathbf{x}_{b}}$ is acute. If the mesh contains obtuse angles, then the method has to be adapted using the unfolding step presented in [15].

\subsection{Construction of subdomains}

The subdomains $\left\{\Omega_{j}\right\}_{j}$ of $\Omega$ are constructed using the FMM. This construction is performed in two steps. The first one consists in introducing master points which are defined as the points which will be the centers of the subdomains. The second one consists in generating the subdomains using these master points as starting points.

(i) Selection of the master points

In the context of the present developments, we consider structures which exhibit stiff parts and flexible parts. The master points are then chosen on the stiff parts. The master points are chosen "uniformly distributed" on the structure and such that the distance between two neighbouring masterpoints is of the order of the smallest wavelength of local displacements that we want to filter. The choice of the master points should be fixed during the step of construction of the computational model with the help of the Computer Aided Design and information given by Engineering.

(ii) Generation of subdomains

To construct the subdomains $\left\{\Omega_{j}\right\}_{j}$ using a set of master points, the fronts starting from the master points are simultaneously propagated until all the nodes become alive nodes. Then, the boundaries of the generated subdomains correspond to the meeting lines of the fronts. 
7. Application to the computational model of an automotive vehicle

In this section, one applies the methodology to construct a G-ROM of an automotive vehicle for which the RN-CM is available.

\subsection{Description of the RN-CM}

The mesh of the RN-CM is made up of 250, 000 nodes and contains various types of finite elements such as volume, surface and beam elements. There are 1, 462, 698 degrees of freedom. The finite element model is shown in Fig. 4. The structure has stiff parts (the frame) and flexible parts (the roof, the windshield, etc.). The frequency band of analysis is $\mathcal{B}=] 0,120] \times 2 \pi \mathrm{rad}_{\text {. }}{ }^{-1}$.

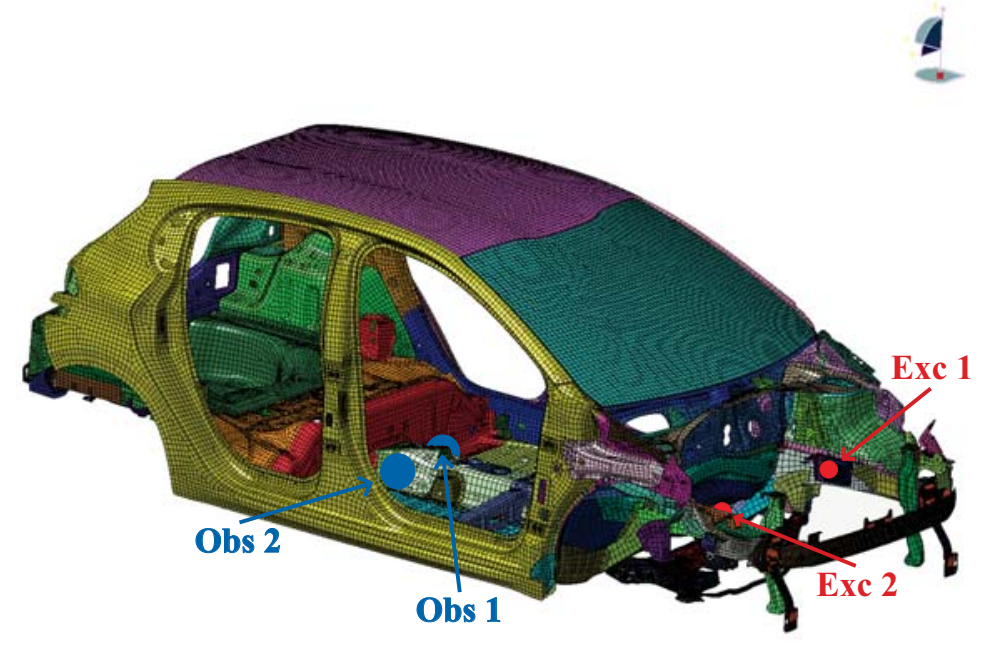

Figure 4: Finite element model of the automotive vehicle. 


\subsubsection{Generation of the subdomains}

The FMM method presented in Section 6 is applied to the mesh of the finite element model of the structure. The number of master points is chosen as $N=90$. Consequently, there will be 90 subdomains. The master points are approximatively uniformly distributed on the stiff part of the structure (see Fig. 5). The subdomains obtained from these master points are represented in Fig. 6.



Figure 5: Master points as the centres of the subdomains.

\subsubsection{Elastic modes, global and local displacements eigenvectors}

The elastic modes are calculated with the FE model. A convergence analysis has been performed to reach convergence in the frequency band of analysis $\mathcal{B}$ yielding $n=160$ elastic modes. The number of elastic modes in frequency band $\mathcal{B}$ is 128 . The first elastic mode $\varphi_{1}$ and the third elastic mode $\varphi_{3}$ are respectively displayed in Fig. 7 and Fig. 8. It can be seen that $\boldsymbol{\varphi}_{1}$ is a local elastic mode while $\boldsymbol{\varphi}_{3}$ is a global one. Some elastic modes are 


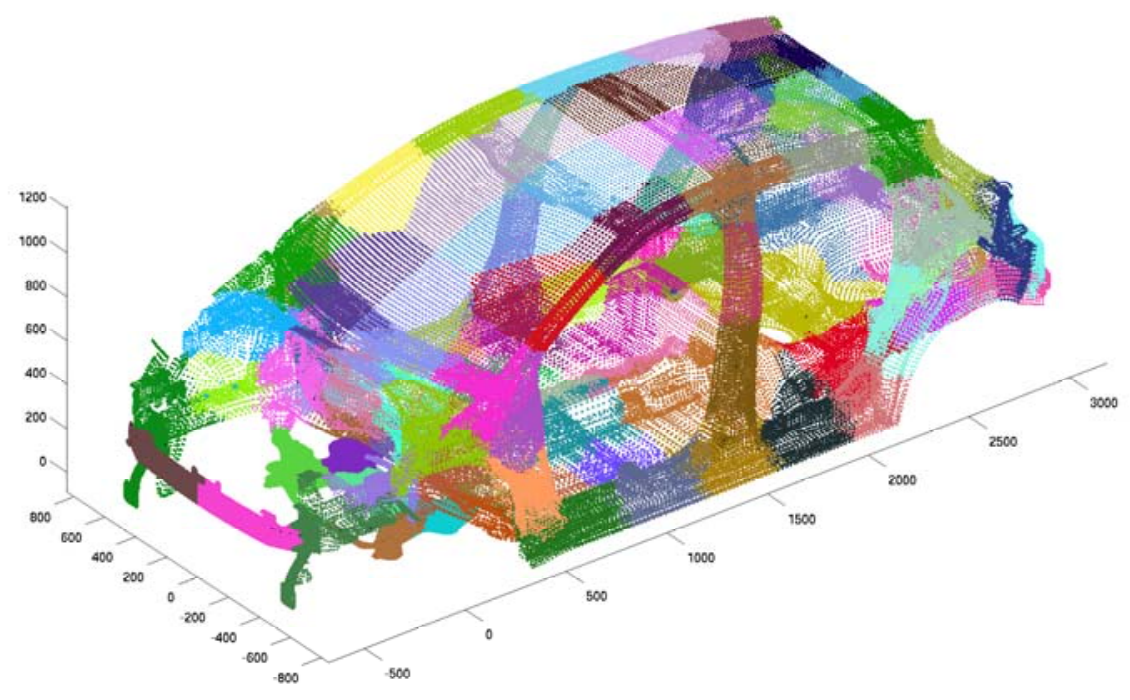

Figure 6: subdomains generated (one per color).

composed of global and local displacements. For example, the $11^{\text {th }}$ elastic mode $\boldsymbol{\varphi}_{11}$, which is displayed in Fig. 9, is a global elastic mode with a high local displacement in the roof.

The global and local displacements eigenvectors are constructed using the double projection method presented in Section 4.2. In the frequency band ] $0,120] \mathrm{Hz}$, there are $n_{g}=36$ global displacements eigenvectors and $n_{\ell}=124$ local displacements eigenvectors. The fourth global displacements eigenvector is plotted in Fig. 10. One can see that this global displacements eigenvector is similar to the $11^{\text {th }}$ elastic mode but the local displacements contributions has been filtered. 


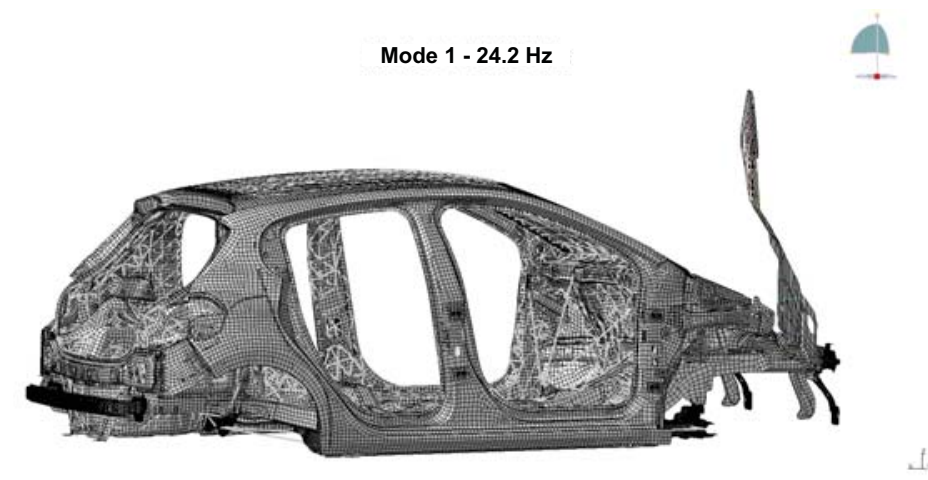

Figure 7: First elastic mode (mode 1 at $24.2 \mathrm{~Hz}$ ).

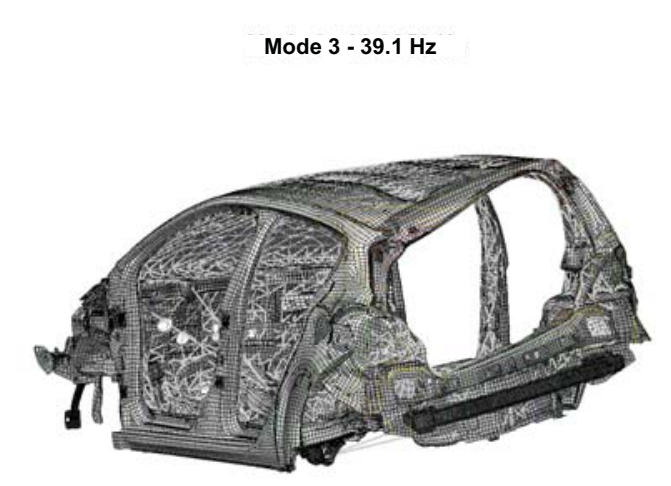

Figure 8: Thirst elastic mode (mode 3 at $39.1 \mathrm{~Hz}$ ).

\subsection{Deterministic frequency response functions}

For all $\omega$ in $\mathcal{B}$, the structure is subjected to two externals point loads equal to $1 N$ applied in each direction $O X$ and $O Z$ to nodes Exc 1 and Exc 2 located in a stiff part (see Fig. 4).

The observations are Obs 1 and Obs 2 which are master points (consequently, are on stiff parts) and which are represented in Fig. 4. The responses are calculated for the different projections associated with the following vectors 


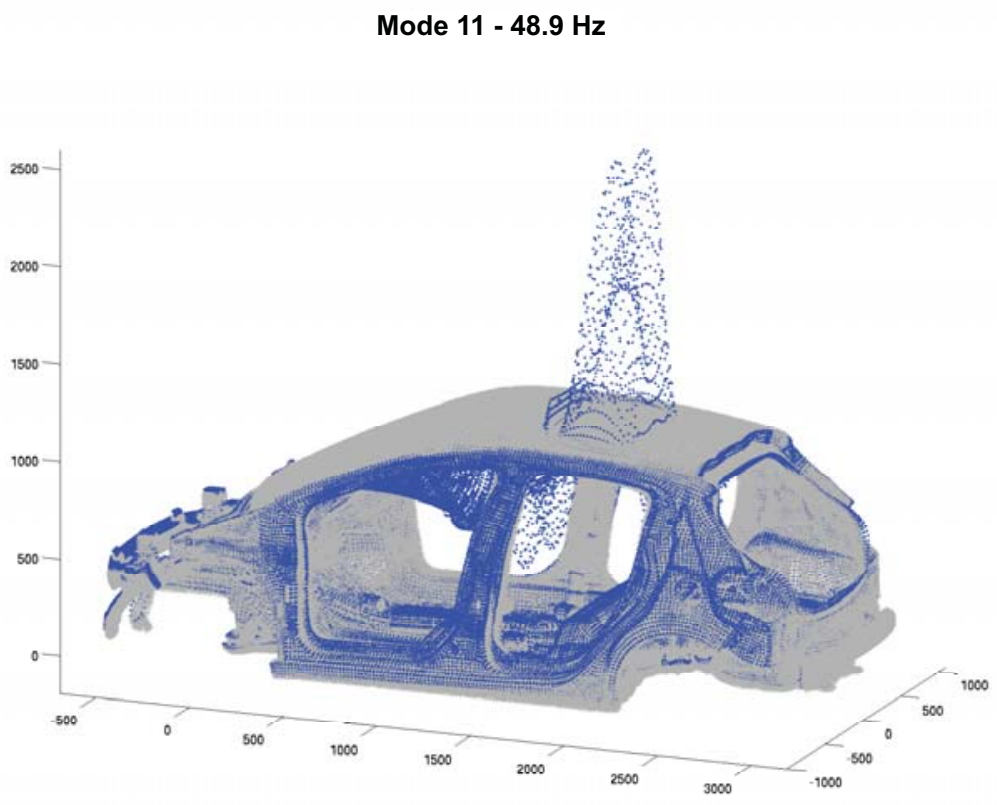

Figure 9: Eleventh elastic mode (mode 11 at $48.9 \mathrm{~Hz}$ ) with global displacements and presenting local displacements on the roof.

bases: (1) with the RN-CM and its approximation constructed with the RNROM presented in Section 3 using the elastic modes ( $\mathbb{U}, n=160)$, (2) with the G-ROM constructed with the global displacements vectors basis defined by Eqs. (16) and (17) using only global displacements eigenvectors $\left(\mathbb{U}^{\left(n_{g}\right)}\right.$ , $n_{g}=36$ and $n_{\ell}=0$ ), (3) with the modified G-ROM constructed with the global displacements vectors basis and the modified damping, defined by Eqs. (20) and (21) using only global displacements eigenvectors $\left(\mathbb{U}_{\text {mod }}\right.$, $n_{g}=36$ and $n_{\ell}=0$ ), and for which the optimal value $\mathbf{a}^{\text {opt }}$ of a has been calculated by Eq. (19), and finally, (4) with the reduced computational model defined by Eq. (15) using the global and the local displacements eigenvec- 


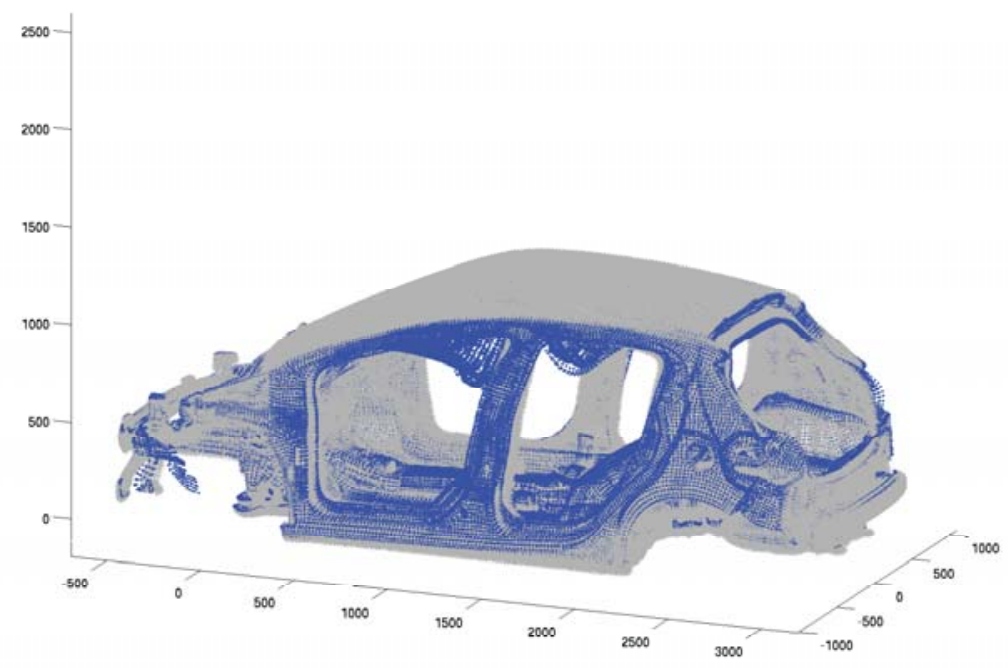

Figure 10: Fourth global displacements eigenvector (fourth eigenvalue 4 at $44.7 \mathrm{~Hz}$ ) corresponding to the eleventh elastic mode (see Fig. 9) for which local displacements on the roof have been filtered.

tors $\left(\mathbb{U}^{\left(n_{g}, n_{\ell}\right)}, n_{g}=36\right.$ and $\left.n_{\ell}=124\right)$. The modulus in $\log _{10}$-scale of the displacements following $O Z$ (vertical direction on the figures) are displayed in Fig. 11 and Fig. 12 for nodes Obs 1 and Obs 2 .

It can be seen that the responses calculated using global and local displacements eigenvectors are exactly the same as the response calculated using the elastic modes (reference solution). We can see that the frequency response function calculate with the global displacements eigenvectors only provides a good approximation of the exact frequency response function (calculate with the elastic modes). The correction improves the responses of the reduced 


\section{Obs 1}

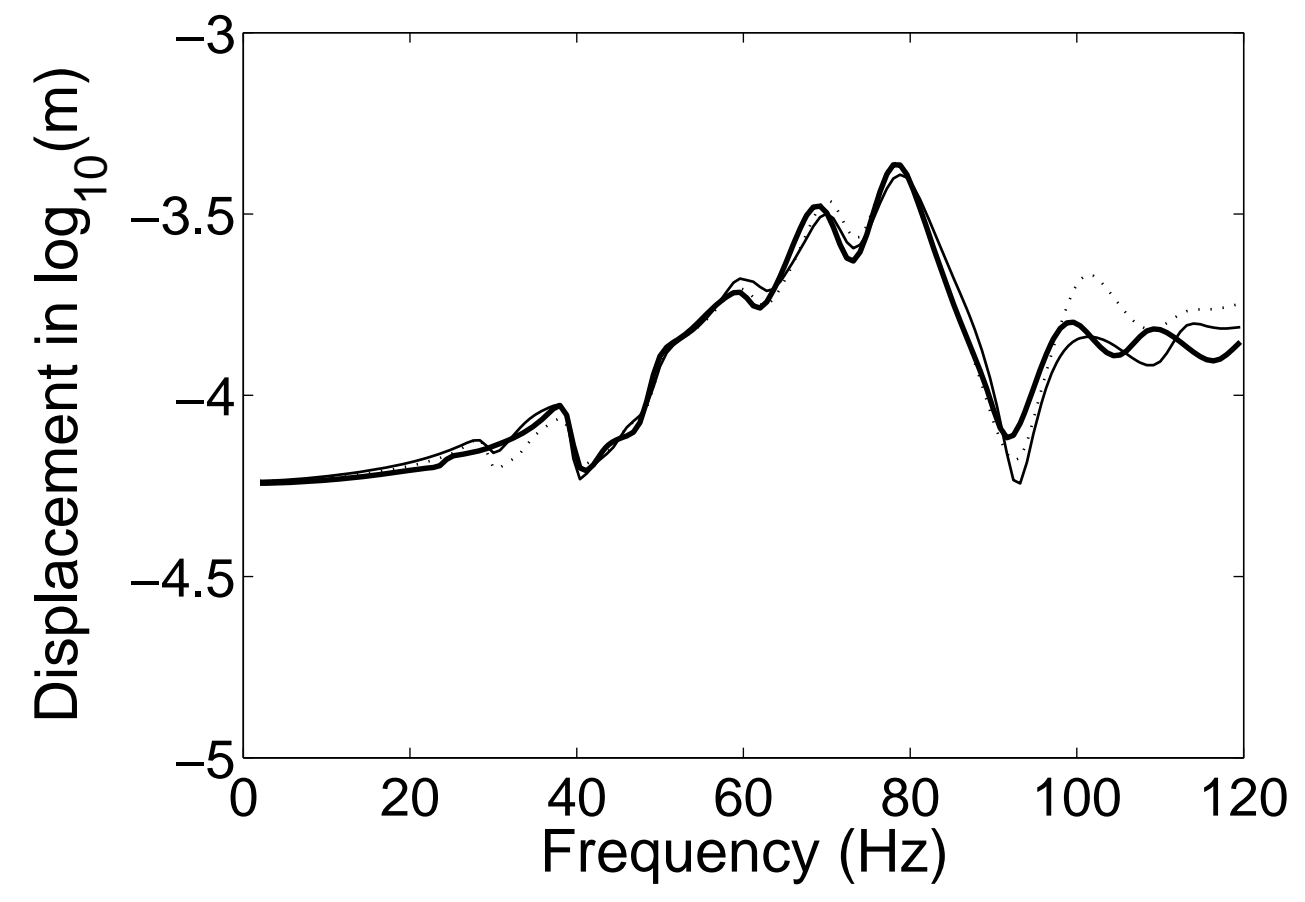

Figure 11: Modulus in $\log _{10}$-scale of the frequency response function for observation, Obs 1. Comparisons between different projection bases: elastic modes (thick line - reference solution), global displacements eigenvectors only (mixed line), global displacements eigenvectors only with damping modification (thin line), global and local displacements eigenvectors (solid line superimposed to the thick line).

model.

\subsection{Reference confidence regions}

The reference nominal frequency response functions for which the results have been presented in Section 7.2 have been calculated using Eq. (3) with $n=160)$ and for which the reference nominal computational model has been defined in Section 7.1. The stochastic reference computational model is 
Obs 2

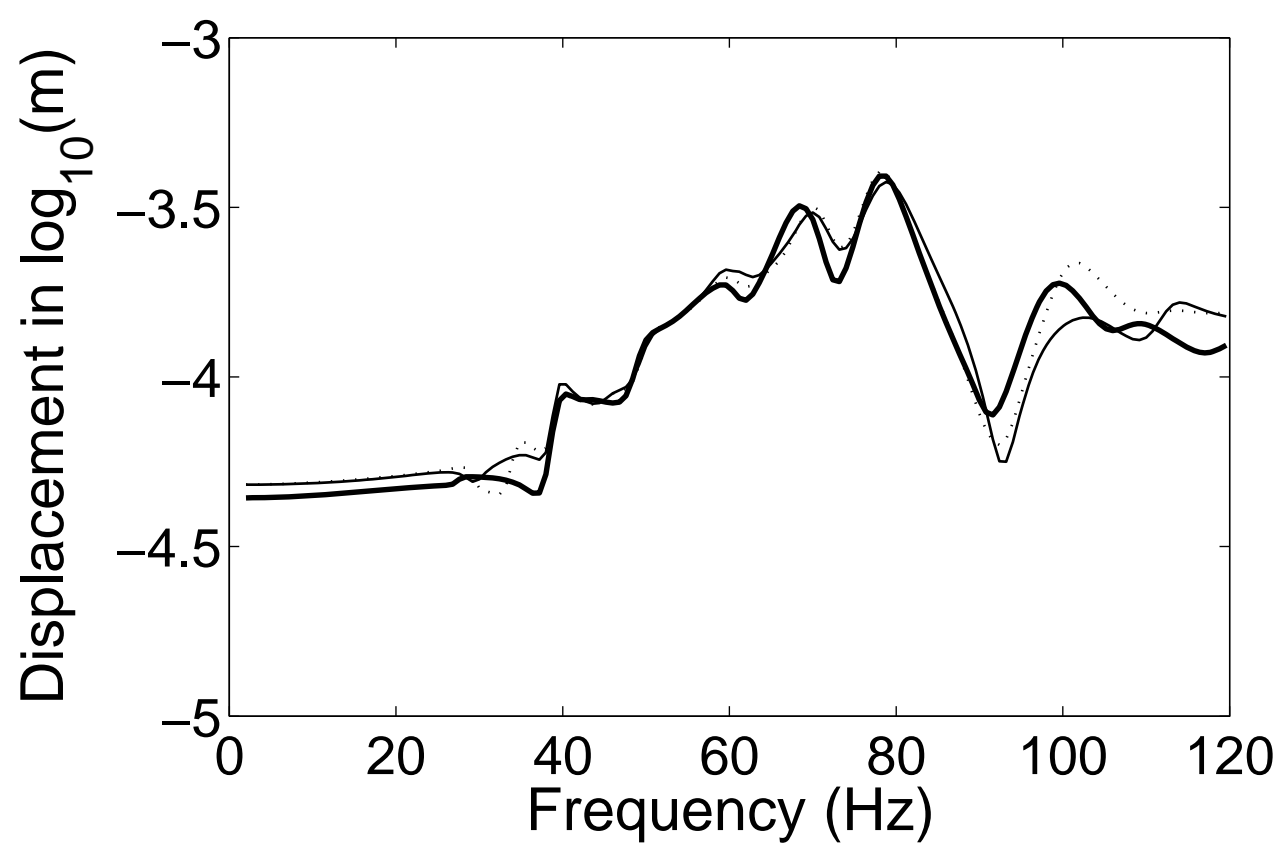

Figure 12: Modulus in $\log _{10}$-scale of the frequency response function for observation, Obs 2. Comparisons between different projection bases: elastic modes (thick line - reference solution), global displacements eigenvectors only (mixed line), global displacements eigenvectors only with damping modification (thin line), global and local displacements eigenvectors (solid line superimposed to the thick line).

thus constructed with the above reference nominal computational model and using the non-parametric probabilistic approach of uncertainties as explained in [11]. The values of the dispersion parameters $\delta_{M}^{r e f}$ and $\delta_{K}^{r e f}$ are those identified in [11]. All the calculations are carried out with the Monte Carlo simulation method for which 1,000 independent realisations are used. The confidence regions corresponding to a probability level $\mathrm{Pc}=0.95$ have been calculated and are plotted in Fig. 13 and Fig. 14 (dark grey regions). These 
confidence regions will be called the reference confidence regions.

\subsection{Random frequency response functions calculated with the stochastic G- $R O M$}

The random frequency response functions are calculated with the stochastic G-ROM defined by Eqs. (22) and (23). All the calculations are carried out with the Monte Carlo simulation method for which 1,000 independent realisations are used. The first step consists in calculating the optimal values of the dispersion parameters $\delta_{M^{g g}}^{\mathrm{opt}}$ and $\delta_{K^{g g}}^{\mathrm{opt}}$ using the maximum likelihood method presented in Section 5.2. The 2-D contour plot of the log-likelihood function $\left(\delta_{M^{g g}}, \delta_{K^{g g}}\right) \mapsto \mathcal{L}\left(\delta_{M^{g g}}, \delta_{K^{g g}}\right)$ is displayed in Fig. 15. The maximum of the log-likelihood function is located in the figure by the coordinates of the optimal point $\delta_{M^{g g}}^{\mathrm{opt}}$ and $\delta_{K^{g g}}^{\mathrm{opt}}$. In a second step, for these optimal values of the dispersions parameters, the confidence regions corresponding to a probability level $\mathrm{Pc}=0.95$ have been calculated and are plotted in Fig. 13 and Fig. 14 (light grey regions).

The validation of the new stochastic G-ROM, which is adapted to the prediction of the frequency responses on the stiff parts in the LF range, can be obtained in comparing the confidence regions constructed with the stochastic G-ROM with the confidence regions constructed with the stochastic RN-CM, for observations on the stiff parts. It is recalled that the solution of the stochastic RN-CM is approximated by the solution of the stochastic RN-ROM (as explained at the end of Section 3). We then have to precise how these confidence regions must be compared in order to perform the validation. The stochastic RN-CM corresponds to the reference which has been experi- 


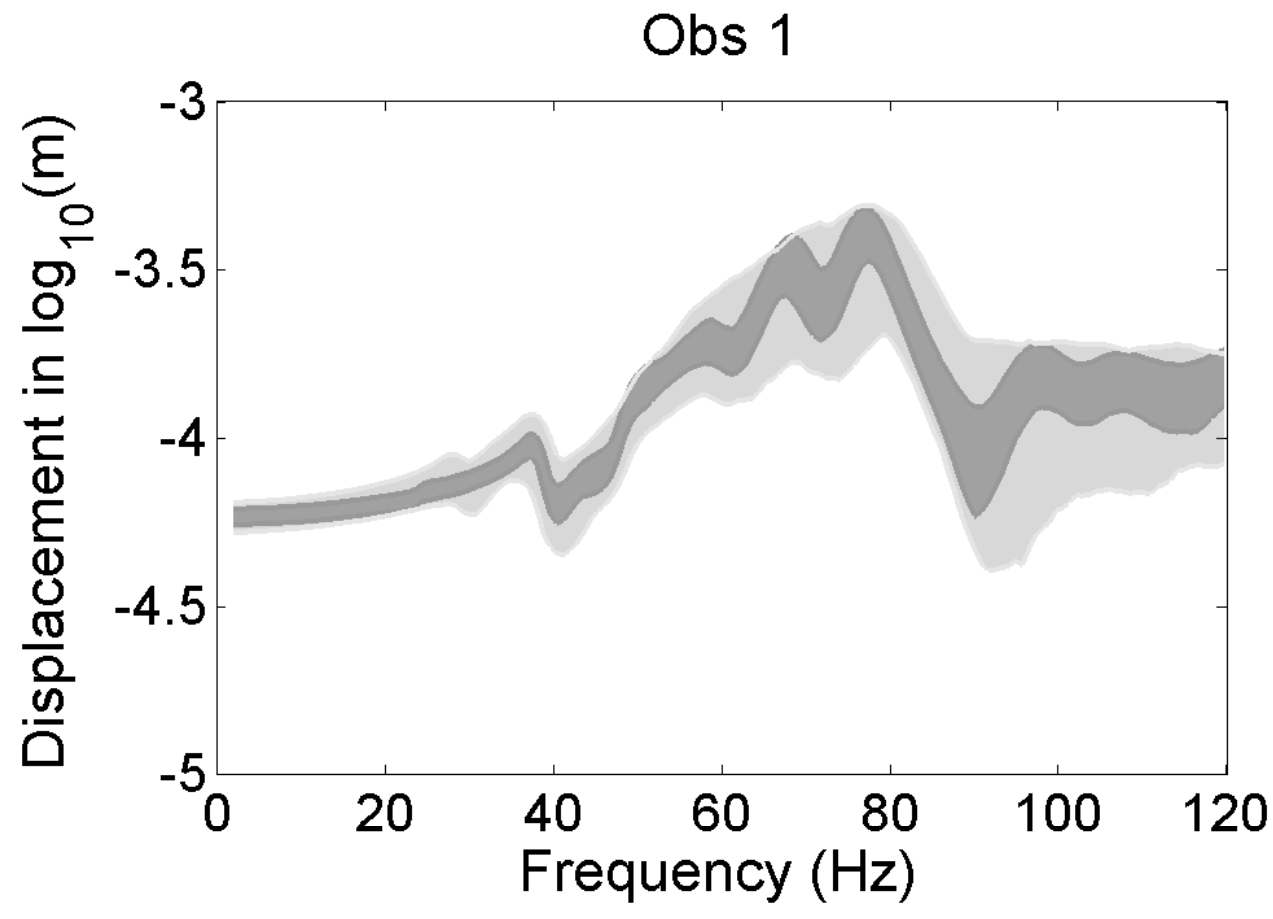

Figure 13: Random frequency response for observation Obs 1. Reference confidence region (dark grey region). Confidence region constructed with the stochastic G-ROM with the global displacements vector basis(light grey region). 
Obs 2

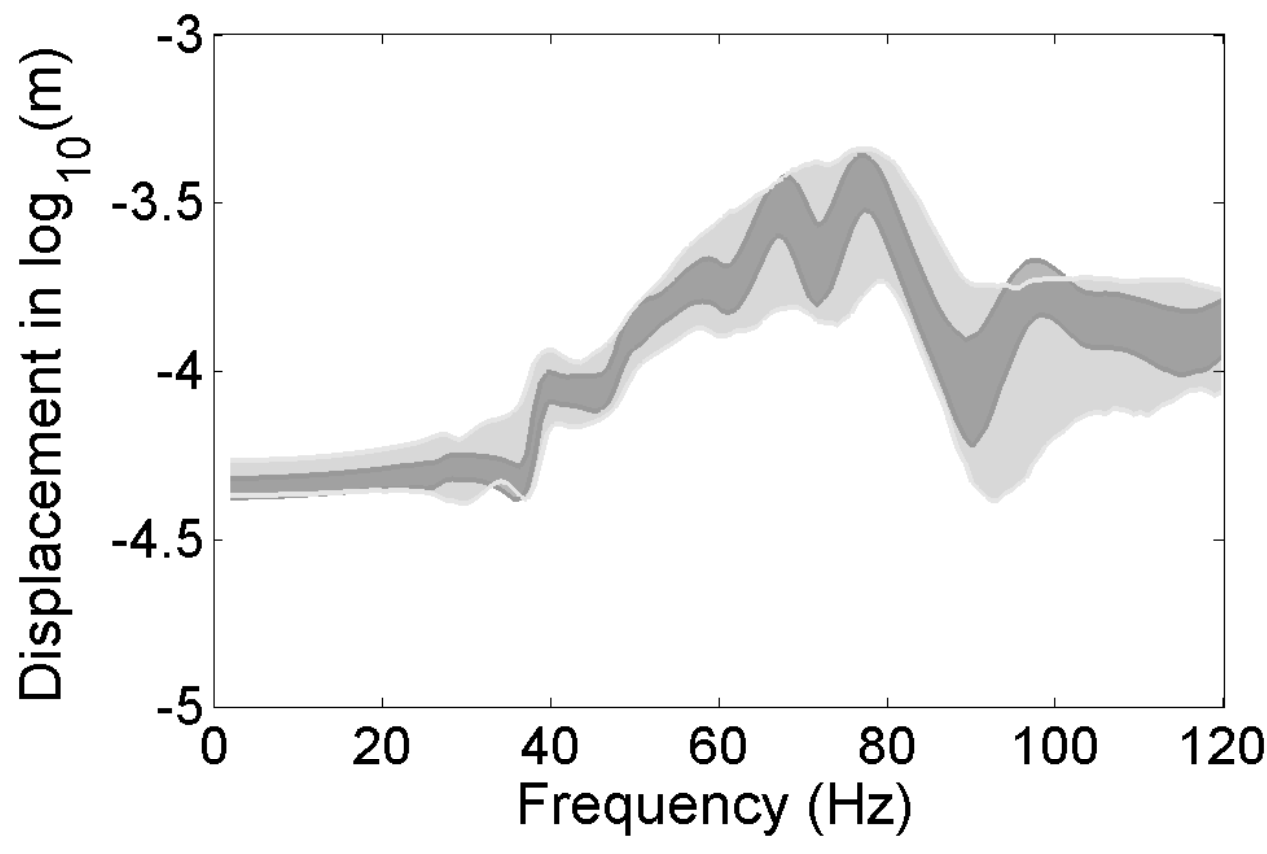

Figure 14: Random frequency response for observation Obs 2. Reference confidence region (dark grey region). Confidence region constructed with the stochastic G-ROM with the global displacements vector basis(light grey region). 


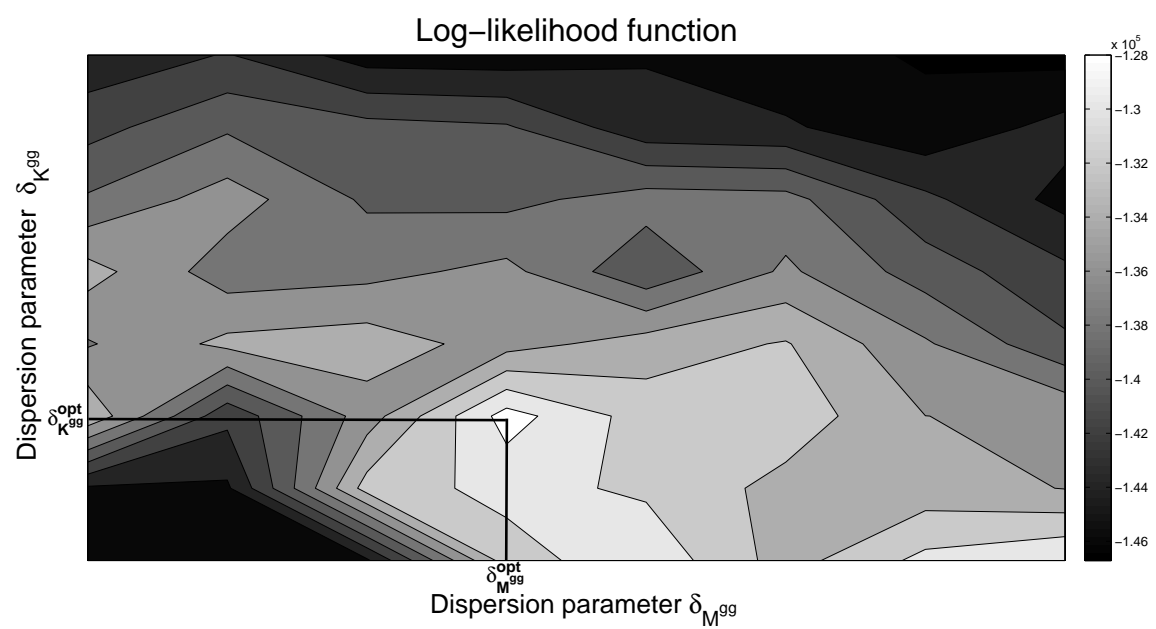

Figure 15: 2-D contour plot of log-likelihood function $\left(\delta_{M^{g g}}, \delta_{K^{g g}}\right) \mapsto \mathcal{L}\left(\delta_{M^{g g}}, \delta_{K^{g g}}\right)$. The optimal values are denoted by $\delta_{M^{g g}}^{\mathrm{opt}}$ and $\delta_{K^{g g}}^{\mathrm{opt}}$.

mentally identified and experimentally validated in $[11,12]$. In the G-ROM, there is an additional modeling error (with respect to the RN-CM) induced by the projection which is performed only on the global displacements eigenvectors (the local displacements contributions for the prediction of the responses on the stiff part, in the LF range, are neglected). Consequently, the level of uncertainties is larger in the G-ROM than in the RN-ROM and therefore, the confidence regions predicted by the stochastic G-ROM must be larger than the confidence regions predicted by the stochastic G-ROM. The validation is obtained if, for each observation, the confidence region computed with the stochastic G-ROM is included in the confidence region computed with the stochastic RN-CM, for most of the frequencies of band $\mathcal{B}$, that is the case. 


\section{Conclusion}

In this paper, we have validated an approach which allows a stochastic G-ROM to be constructed in order to predict the responses on the stiff parts, and in the low frequency domain, of a complex structure having numerous local elastic modes which cannot easily be separated from the global elastic modes with the usual reduction methods. This validation has been performed for a very complex dynamical system consisting in an automotive vehicle and includes the validation of the Fast Marching Method which is adapted to complex geometry and which allows the computational subdomains to be constructed.

\section{References}

[1] Y. Hahn, N. Kikuchi, Identification of global modeshape from a few nodal eigenvectors using simple free-form deformation, Engineering with Computers, 21(2) 115-128.

[2] R. Ohayon, C. Soize, Structural acoustics and vibration, Academic press, 1998.

[3] S.K. Hong, B.I. Epureanu, M.P. Castanier, D.J Gorsich, Parametric reduced order models for predicting the vibration response of complex structures with component damage and uncertainties, Journal of Sound and Vibration, 330(6)(2011) 1091-1110.

[4] W.H. Shyu, J.M. Gu, G.M. Hulbert, Z.-D Ma, On the use of multiple quasi-static mode compensation sets for component mode synthesis of 
complex structure, Finite Elements in Analysis and Design, 35 (2000) 119-140.

[5] M.C.C. Bampton, R.R. Craig Jr, Coupling of substructures for dynamic analyses, American Institue of Aeronautics and Astronautics Journal, 6(7) (1968) 1313-1319.

[6] M. Wamsler, On the selection of the mode cut-off number in component mode reduction, Engineering with Computers, 25(2) (2009) 139-146.

[7] S. Donders, R. Hadjit, M. Brughmans, L. Hermans, W. Desmet, A wavebased substructuring approach for fast vehicle body optimisation, International Journal of Vehicle Design, 43(1-4) (2007) 100-115.

[8] C. Soize and A. Batou, Stochastic reduced-order model in low-frequency dynamics in presence of numerous local elastic modes, Journal of Applied Mechanics - Transaction of the ASME, 78(6) (2011) 061003.

[9] C. Soize, A nonparametric model of random uncertainties for reduced matrix models in structural dynamics, Probabilistic Engineering Mechanics, 15(3) (2000) 277-294.

[10] O.C. Zienkiewiez, R.L. Taylor, The Finite Element Method, Butterworth-Heinemann, Oxford, 2000.

[11] J.F Durand, C. Soize, L. Galiardini, Structural-acoustic modeling of automotive vehicles in presence of uncertainties and experimental identification and validation, Journal of the Acoustical Society of America, $124(3)$ (2008) 1513-1525. 
[12] C. Fernandez, C. Soize, L. Galiardini, Sound-insulation layer modeling in car computational vibroacoustics in the medium-frequency range, Acta Acustica united with Acustica (AAUWA), 96(3) (2010) 437-444.

[13] M. Kassem, C. Soize, L. Galiardini, Structural partitioning of complex structures in the medium-frequency range. An application to an automotive vehicle, Journal of Sound and Vibration, 330(5) (2011) 937-946.

[14] J.A. Sethian, A Fast Marching Level Set Method for Monotonically Advancing Fronts, Proceedings of the National Academy of Sciences, 93(4) (1996) 1591-1595.

[15] J.A. Sethian, R. Kimmel, Computing Geodesic Paths on Manifolds, Proceedings of the National Academy of Sciences, 95 (1998) 8431-8435. 


\section{List of figure captions}

Figure 1: Simple case: spatial discretisation for the Eikonale equation.

Figure 2: Diagram of the Fast Marching Method: step 1 (a), step 2 (b), step 3 (c) and last step corresponding to the diagram, step 5 (d).

Figure 3: Triangular mesh (with acute angle) around node $\mathbf{x}_{c}$.

Figure 4: Finite element model of the automotive vehicle.

Figure 5: Master points as the centres of the subdomains.

Figure 6: subdomains generated (one per color).

Figure 7: First elastic mode (mode 1 at $24.2 \mathrm{~Hz}$ ).

Figure 8: Thirst elastic mode (mode 3 at $39.1 \mathrm{~Hz}$ ).

Figure 9: Eleventh elastic mode (mode 11 at $48.9 \mathrm{~Hz}$ ) with global displacements and presenting local displacements on the roof.

Figure 10: Fourth global displacements eigenvector (fourth eigenvalue 4 at $44.7 \mathrm{~Hz}$ ) corresponding to the eleventh elastic mode (see Fig. 9) for which local displacements on the roof have been filtered. 
Figure 11: Modulus in $\log _{10}$-scale of the frequency response function for observation, Obs 1. Comparisons between different projection bases: elastic modes (thick line - reference solution), global displacements eigenvectors only (mixed line), global displacements eigenvectors only with damping modification (thin line), global and local displacements eigenvectors (solid line superimposed to the thick line).

Figure 12: Modulus in $\log _{10}$-scale of the frequency response function for observation, Obs 2. Comparisons between different projection bases: elastic modes (thick line - reference solution), global displacements eigenvectors only (mixed line), global displacements eigenvectors only with damping modification (thin line), global and local displacements eigenvectors (solid line superimposed to the thick line).

Figure 13: Random frequency response for observation Obs 1. Reference confidence region (dark grey region). Confidence region constructed with the stochastic reduced order model with the global displacements vector basis(light grey region).

Figure 14: Random frequency response for observation Obs 2. Reference confidence region (dark grey region). Confidence region constructed with the stochastic reduced order model with the global displacements vector basis(light grey region).

Figure 15: 2-D contour plot of log-likelihood function $\left(\delta_{M^{g g}}, \delta_{K^{g g}}\right) \mapsto \mathcal{L}\left(\delta_{M^{g g}}, \delta_{K^{g g}}\right)$. 
The optimal values are denoted by $\delta_{M^{g g}}^{\mathrm{opt}}$ and $\delta_{K^{g g}}^{\mathrm{opt}}$. 